\title{
Effectiveness of assistive torque patterns supplied by a pelvis exoskeleton after slippages: a pilot study
}

\author{
F. Aprigliano*, V. Monaco*, P. Tropea, D. Martelli, N. Vitiello, S. Micera
}

\begin{abstract}
Falls are acknowledged as one of the most serious causes of health problems in the elderly population. Thus, there is an urgent need to develop novel technological solutions to counteract the balance loss and prevent falls. In this pilot study, we enrolled two healthy older volunteers to investigate the effectiveness of different assistive torque patterns provided by a pelvis exoskeleton in promoting balance recovery after slippages. Our results revealedthat the best strategy to counteract the balance loss after a slippage consists in slowing the slipping foot and minimizing the downward movement of the center of mass by providingopposite torque patterns between hips.
\end{abstract}

\section{INTRODUCTION}

$\mathrm{F}_{\mathrm{c}}$ ALLS are one of the most serious problems in the elderly [1]. Counteracting the risk of fall is hence of primary importance to allow the growing elderly population to gain safety and autonomy during their daily activities.

To address this issue, we have recently tested the effectiveness of a closed-loop strategy combining a predictive algorithm able to detect signs of balance loss due to a slippage, and a wearable robotic exoskeleton supplying an assistive strategy (Fig. 1;[2]).The robot-mediated strategy was empirically defined in accordance with physiological countermeasure described in literature[3], thus the exoskeleton synchronously promoted hip extension of the perturbed limb (PL) and hip flexion ofcontralateral one (unperturbed limb; UL).

Despite its effectiveness, duration and intensity of the assistive torques are two critical issues which have never been investigated before. As a matter of fact, from the best of our knowledge, no authors have ever closed the loop from detecting an incipient fall to provide a suitable support by means of a wearable robotic platform.

In this pilot study, we tested the hypothesis that other torque patterns could be effective in promoting the balance recovery after unexpected slippages. Alternative counteractions were obtained by differently combining synchronous/phase-shifted and flex/extensors patterns

This work was supported by the European Union within the CYBERLEGs and the CYBERLEGs Plus Plus projects.

* authors equally contributed and alphabetic order.

F. Aprigliano, V. Monaco, N. Vitiello and S. Micera are with the BioRobotics Institute, ScuolaSuperioreSant'Anna, Pisa, Italy (corresponding author to provide e-mail: vito.monaco@santannapisa.it).P. Tropea is with Casa di Cura del Policlino di Milano, Italy. D. Martelli is with Columbia University, New York, USA. S. Micera is also with EPFL, Lausanne, Switzerland. between the two limbs (Fig. 2).

\section{MATERIALS AND METHODS}

\section{A. Participants and experimental protocol}

Two healthy elderly subjects(69.5 \pm 6.4 years old) were asked to manage unexpected slippages while being assisted by theActive Pelvis Orthosis (APO), as detailed elsewhere (Fig. 1; [2]). Full body kinematics was recorded at $250 \mathrm{~Hz}$ using a six camera-based system (Vicon, Oxford, U.K.). Perturbations of the steady walking were delivered by a custom platform [4] and consisted in unexpected slippages along the Antero-Posterior (AP) direction.

The APO was designed to assist hip flexion-extension in the sagittal plane[5] and was set to either be transparent during the whole experimental test (Z-mode)or to supply suitable torque patterns at both hips to promote stability in case of balance loss. Concerning the latter strategy, in our previous study we demonstrated the effectiveness of a synchronous couple of extensor and flexor torque patterns at PL and UL, respectively, named A-mode [2]. In this study, we investigated the effectiveness of three different torques patterns accounting for: (1) asynchronous flexor torque for the PL and extensor torque for the UL with a time shift of $100 \mathrm{~ms}$ (named A1; Fig. 2a); (2)synchronous extensor patterns for both limbs (named A2; Fig. 2b); (3)asynchronous extensor patters for both limbs with a time shift of $100 \mathrm{~ms}$ (named A3; Fig. 2c).

Research procedures were in accordance with the Declaration of Helsinki and were approved by the Institutional Ethics Committee of Don Gnocchi Foundation (Firenze, Italy), where the experiments occurred.

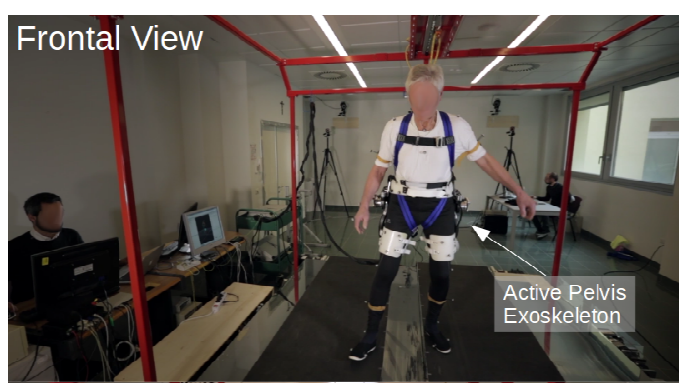

Fig. 1. An elderly volunteer was wearing the Active Pelvis Orthosis while managing a slippage provided by the mechatronic platform during walking.

\section{B. Data processing}

Marker trajectories were low-pass filtered with a cut-off frequency of $10 \mathrm{~Hz}$, using a $4^{\text {th }}$ order, zero-lag Butterworth filter. A 15-segment biomechanical model was developed. Body segment inertial parameters (i.e., mass and centre of 
mass (CoM) position) and joint centre locations were estimated according to [6]. Gait events were identified by visual inspection of the kinematics of the feet and the vertical component of the ground reaction force.

\section{Dynamic stability}

To investigate the effectiveness of the proposed assistive strategies to promote balance recovery after slippages, the following metrics were calculated: Margin of Stability (MoS; [7]) and CoM stability[8], [9].Moreover, the "CoM motion state" was used to investigate the ongoing of the balance recovery. Briefly, a stability region was identified as a diagonal band in the horizontal CoM velocity-position phase plane, located both inside and outside the base of support (BoS) (Fig. 2; [8], [9]).
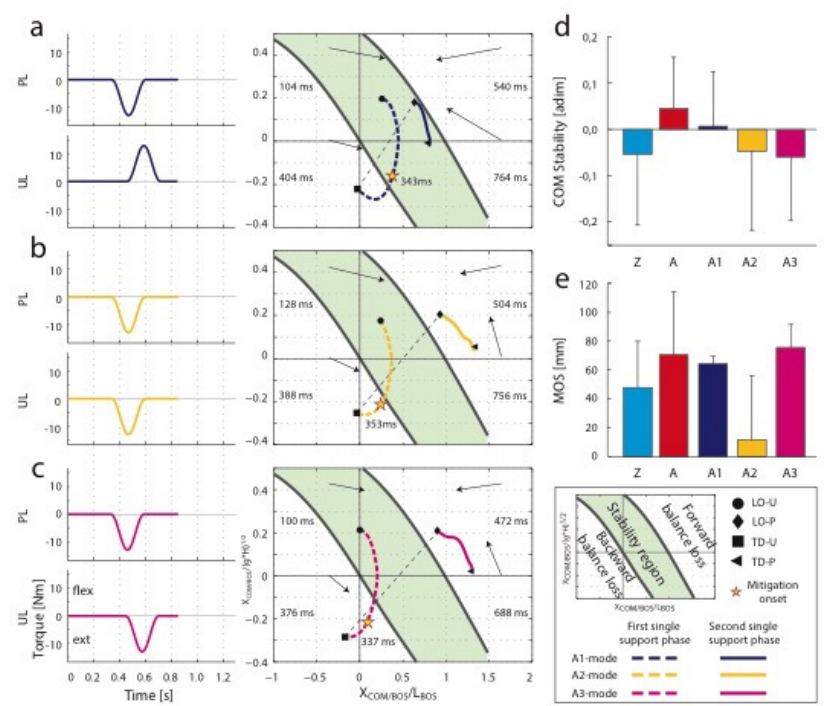

Fig. 2. (a-c) The CoM motion state of one elder is shown during A1-, A2- and A3- modes, respectively (purple, orange and magenta lines). Four consecutive time events were identified, after the perturbation onset: (I) the lift off of the unperturbed foot $\left(\mathrm{LO}_{\mathbf{U}}\right)$; (II) the instant immediately before (i.e., 1 frame, consisting in $1 / 250 \mathrm{~s}$ ) the touch down of the unperturbed foot $\left(\mathrm{TD}_{\mathbf{U}}\right)$; (III) the lift off of the perturbed foot $\left(\mathrm{LO}_{\mathbf{P}}\right)$; and (IV) the instant immediately before the touch down of the perturbed foot $\left(\mathrm{TD}_{\mathrm{P}}\right) . \mathrm{CoM}$ stability (panel d) and Margin Of Stability (MoS; panel e) are reported for $\mathrm{Z}$ - and $\mathrm{A}_{-}, \mathrm{A}_{1^{-}}, \mathrm{A}_{2}$ - and $\mathrm{A}_{3}$-modes and for both elders (mean values $\pm \mathrm{SD}$ ).

\section{RESULTS}

The time course of the "CoM motion state" is shown for three assistive strategies supplied on an older adult, as representative case (Fig. 2). In particular, during the first single support phase (I-II events, $\mathrm{LO}_{\mathrm{U}}-\mathrm{TD}_{\mathrm{U}}$, dashed lines), intra-subjects differences were not observed between A1-, A2- and A3-modes, since the assistive strategy was not yet enabled. Conversely, during the second single support phase (III-IV events, $\mathrm{LO}_{\mathrm{P}}-\mathrm{TD}_{\mathrm{P}}$, full lines), the "CoM motion state" changed according to the APO working conditions. Specifically, the "CoM motion state" at the end of the compensatory stride (i.e., $\mathrm{TD}_{\mathrm{P}}$ ) was within or above to the limit of the forward balance loss region, if APO worked in
A1- or A2- and A3-modes, respectively.

On the whole, the CoM stability and the MoS calculated across elderly subjects during A1-, A2- and A3-modes were always lower than those observed during the A-mode (Fig. $3 \mathrm{~d}$ and $3 \mathrm{e}$, respectively). Specifically, the CoM stability for the strategies A2 and A3 was worse than for A and was comparable to the CoM stability achieved without assistance (Z). On the other side, a slight shift of opposed torque patterns (strategy A1) allowed the CoM motion state, averaged across all participants, to falls just within the stability region at the end of the observed time window $\left(\mathrm{TD}_{\mathrm{P}}\right)$.

\section{CONCLUSION}

Results revealed that the alternative strategies tested in this pilot study (A1-, A2-, A3-mode) are not effective as the one previously assessed(A-mode;[2]). Specifically, our results confirmed that the best strategy to counteract the lack of balance after a slippage consists in slowing the slipping foot and, possibly, minimizing the downward movement of the center of mass by using opposite torque patterns between hips, as described above. In this respect, although the A1mode slightly resembles the A-mode[2], the delay between the limbs deteriorates the effectiveness of the counteracting action.

On the other hand, different torque patterns (A2- and A3mode) are likely non-useful with respect to the adopted perturbation paradigm. Accordingly, further analysis will not involye these control strategies.

\section{REFERENCES}

T. Masud and R. O. Morris, "Epidemiology of falls," Age Ageing, vol. 30, no. SUPPL. 4, pp. 3-7, 2001.

V. Monaco, P. Tropea, F. Aprigliano, D. Martelli, A. Parri, M. Cortese, R. Molino-Lova, N. Vitiello, and S. Micera, "An ecologically-controlled exoskeleton can improve balance recovery after slippage," Sci. Rep., vol. 7, no. March, p. 46721, 2017.

B. E. Moyer, M. S. Redfern, and R. Cham, "Biomechanics of trailing leg response to slipping - Evidence of interlimb and intralimb coordination," Gait Posture, vol. 29, no. 4, pp. 565-570, 2009.

L. Bassi Luciani, V. Genovese, V. Monaco, L. Odetti, E. Cattin, and S. Micera, "Design and Evaluation of a new mechatronic platform for assessment and prevention of fall risks," $J$. Neuroeng. Rehabil., vol. 9, no. 1, p. 51, 2012.

F. Giovacchini, F. Vannetti, M. Fantozzi, M. Cempini, M. Cortese, A. Parri, T. Yan, D. Lefeber, and N. Vitiello, "A lightweight active orthosis for hip movement assistance," in Robotics and Autonomous Systems, 2015, vol. 73, pp. 123-134.

D. Martelli, V. Monaco, L. Bassi Luciani, and S. Micera, "Angular momentum during unexpected multidirectional perturbations delivered while walking," IEEE Transactions on Biomedical Engineering, vol. 60, no. 7, pp. 1785-1795, 2013.

] A. L. Hof, "The 'extrapolated center of mass' concept suggests a simple control of balance in walking," Hum. Mov. Sci., vol. 27, no. 1, pp. 112-125, 2008.

Y. C. Pai and J. Patton, "Center of mass velocity-position predictions for balance control," J. Biomech., vol. 30, no. 4, pp. 347-354, 1997.

F. Yang, F. C. Anderson, and Y. C. Pai, "Predicted threshold against backward balance loss following a slip in gait," $J$. Biomech., vol. 41, no. 9, pp. 1823-1831, 2008. 